\title{
PREDICTING MIGRATORY FLIGHT ALTITUDES BY PHYSIOLOGICAL MIGRATION MODELS
}

\author{
Felix LieChti, ${ }^{1,3}$ MARCEl KlaAssen, $^{2}$ AND BRUNO BRUdereR ${ }^{1}$ \\ ${ }^{1}$ Swiss Ornithological Institute, $\mathrm{CH}-6204$ Sempach, Switzerland; and \\ ${ }^{2}$ Center for Limnology, Netherlands Institute of Ecology, Rijksstraatweg 6, NL-3631 Nieuwersluis, The Netherlands
}

\begin{abstract}
Using the altitudinal profiles of wind, temperature, pressure, and humidity in three flight models, we tried to explain the altitudinal distributions of nocturnal migrants recorded by radar above a desert in southern Israel. In the simplest model, only the tailwind component was used as a predictor of the most preferred flight altitude (T model). The energy model (E model) predicted flight ranges according to mechanical power consumption in flapping flight depending on air density and wind conditions, assuming optimal adjustment of airspeed and compensation of crosswinds, and including the influence of mass loss during flight. The energy-water model (EW model) used the same assumptions and parameters as the E model but also included restrictions caused by dehydration. Because wind was by far the most important factor governing altitudinal distribution of nocturnal migrants, differences in predictions of the three models were small. In a first approach, the EW model performed slightly better than the E model, and both performed slightly better than the T model. Differences were most pronounced in spring, when migrants should fly high according to wind conditions, but when climbing and descending they must cross lower altitudes where conditions are better with respect to dehydration. A simplified energy model (Es model) that omits the effect of air density on flight costs explained the same amount of variance in flight altitude as the more complicated E and EW models. By omitting the effect of air density, the Es model predicted lower flight altitudes and thus compensated for factors that generally bias height distributions downward but are not considered in the models (i.e. climb and descent through lower air layers, cost of ascent, and decrease of oxygen partial pressure with altitude). Our results confirm that wind profiles, and thus energy rather than water limitations, govern the altitudinal distribution of nocturnal migrants, even under the extreme humidity and temperature conditions in the trade wind zone. Received 8 October 1998, accepted 8 July 1999.
\end{abstract}

LONG-DISTANCE FLIGHTS over wide ecological barriers are common for many migrating birds (Moreau 1972, Williams and Williams 1990). Cost, duration, and distance covered per flight stage depend on flight capacities of the birds and atmospheric conditions en route. A single flight may be restricted by energy expenditure or by water loss (Carmi et al. 1992). Although general principles of energy expenditure during flight are fairly well understood (Pennycuick 1989, Rayner 1990), little is known about the physiological components that govern flight behavior of migrating birds (Klaassen 1996), and empirical data on the choice of migrating birds for particular atmospheric conditions are scarce (Bruderer et al. 1995).

Carmi et al. (1992) developed a model to predict maximum flight ranges with respect to energy and water loss according to a bird's pre-

\footnotetext{
${ }^{3}$ E-mail: liechtif@orninst.ch
}

migratory condition, flight capacity, and the atmospheric situation. This model suggests that dehydration rather than energy will limit flight range and that due to a decrease in oxygen partial pressure with altitude (causing increased respiratory ventilation), birds should fly at altitudes below $1,000 \mathrm{~m}$ to cross the Sahara successfully. Although Carmi et al. (1992) neglected the influence of wind on flight range, Bruderer et al. (1995) predicted a high percentage of the altitudinal distribution of bird migration with a mechanistic model that was based only on the altitudinal profile of the tailwind component (including headwinds as negative tailwinds). Klaassen (1996) proposed a combination of wind influence and water/energy constraints. Precise radar measurements of height distributions of nocturnal migrants over southern Israel in autumn and spring (Bruderer and Liechti 1995, Bruderer et al. 1995), combined with simultaneous recordings of altitudinal profiles of weather variables, offered the op- 
portunity to validate the presumed importance of wind conditions, potential energy, and water constraints on flight range.

Our study deals with flight ranges calculated according to the models suggested by Klaassen (1996) that incorporate energy constraints including wind influence, and energy and water constraints including wind influence (see below). These physiological models were put into context with the predictive value of the tailwind component alone according to Bruderer et al. (1995). Applying the three models to the altitudinal profiles of meteorological factors measured in southern Israel, we predicted flight ranges for various flight altitudes. Variation in flight range with altitude was used as an index of suitability of these altitudes for migration. This suitability index was compared with the altitudinal distribution of nocturnal migrants as determined by radar. A simplified energy model, including optimal flight behavior with respect to wind, but neglecting a possible change in flight behavior as a result of decreasing air pressure with altitude, eventually was used in a comparable manner to make predictions on flight altitude.

\section{METHODS}

The models._Bruderer et al. (1995) presented a mechanistic model for predicting flight altitude based on a specific searching behavior for altitudes with good tailwind components. Based on this model, we established an index of suitability for flight conditions that included only the tailwind component, which was calculated as wind speed times the cosine of the wind direction relative to the migratory direction (thus neglecting the possibility that birds compensate for wind drift due to crosswinds). This model does not take into account any adaptation of flight behavior with respect to the physiology of the bird. We refer to this model as the T model. The energy model (E model) predicts flight ranges based on Pennycuick's (1989) model for mechanical flight power in flapping flight. Predictions for flight range depend on energy reserves, energy expenditure, and tailwind support. It is assumed that the bird compensates completely for crosswinds and adjusts airspeed in an energetically optimal way (Liechti et al. 1994). The use of a simplified energy model (Es model) that neglects the expected increase in airspeed with decreasing air pressure is discussed. In the energy-water model (EW model) the water budget is determined by power consumption, air pressure, ambient temperature, and relative humidity, where the calculation of power consumption relies on the same parameters as in the E model. Although in the E model the limit to flight range is imposed by energy availability only, the EW model includes an additional restriction caused by dehydration (see Carmi et al. 1992; Klaassen 1995, 1996). A computer program of the EW model is available in Klaassen et al. (1999).

Model parameters. - To establish tailwinds, headwinds, and crosswinds, mean vectors of the very narrow distribution of track directions of nocturnal migrants under calm conditions were taken as the reference for migratory direction (Liechti and Bruderer 1995). These mean vectors were $190^{\circ}$ in autumn and $360^{\circ}$ (due north) in spring. Predictions were performed for an example of one small (Willow Warbler [Phylloscopus trochilus]) and one large (Golden Oriole [Oriolus oriolus]) passerine, in line with parameters used by Carmi et al. (1992) and Klaassen and Biebach (2000). This size range included more than $80 \%$ of the nocturnal migrants in this area (Liechti and Bruderer 1995). Input data for Willow Warbler and Golden Oriole, respectively, were body mass, 10 and $95 \mathrm{~g}$; wing span, 0.17 and $0.46 \mathrm{~m}$; wing area, 0.0069 and $0.0505 \mathrm{~m}^{2}$; fuel fraction of initial body mass, 0.300 and 0.368; and water fraction of initial body mass, 0.50 and 0.45 (assuming $71 \%$ water content of lean tissue). The maximum water-loss fraction of initial water content was arbitrarily set to a rather conservative value of $30 \%$. The standard settings for the calculation of flight power were taken from Pennycuick's program (1989), except the drag coefficient was altered to 0.08 according to new results from wind-tunnel experiments (Pennycuick et al. 1997). Fuel reserves were considered to consist of $70 \%$ fat, $9 \%$ protein, and $21 \%$ water, resulting in an energy density of $29 \mathrm{~kJ} / \mathrm{g}$ and a metabolic water yield of 1.00 g per gram of fuel (Klaassen 1996, Jenni and JenniEiermann 1998).

Field data.-We collected field data in the Arava Valley, Israel $\left(30^{\circ} 50^{\prime} \mathrm{N}, 35^{\circ} 20^{\prime} \mathrm{E}\right)$, during autumn (12 September to 30 October) 1991 and spring (1 March to 19 May) and early autumn (13 August to 18 September) 1992. The site was located between the Dead Sea and the Gulf of Eilat and Aqaba, $150 \mathrm{~m}$ below sea level, in the Negev portion of the Rift Valley. With a tracking radar of the type "Superfledermaus," intensity and altitudinal distribution of migration were registered every $2 \mathrm{~h}$ up to $6 \mathrm{~km}$ above ground level. Because most migration was below $4 \mathrm{~km}$, and reliability of the measurements decreases at high altitudes, the analysis was restricted to the lowest $4 \mathrm{~km}$.

A calibrated sensitivity time control was applied to eliminate insect echoes at close ranges (Bruderer 1997). Additionally, exceptional echoes from rain clouds or concentrations of insects were interactively marked and excluded from the single measurements (PPI pictures). Heavily contaminated measurements were completely removed from analysis. During the time between the quantitative measurements, data 
on the flight paths and wingbeat patterns of migrants were registered by tracking single targets. Radiosonde balloons launched around midnight provided height profiles of air pressure, air temperature, and relative humidity up to about $5 \mathrm{~km}$ above ground level. Wind measurements were performed every $4 \mathrm{~h}$ by tracking the ascent of a pilot balloon by radar. Processing of all these data (migration density and weather variables) resulted in altitudinal profiles with height intervals of $200 \mathrm{~m}$. Density measurements were averaged from 2300 and 0100 local time (GMT + 2h) for autumn 1991 and spring 1992, and from 2200 and 2400 for autumn 1992. Wind and radiosonde data were always from the full hour between these two measurement. Most (90\%) of the targets tracked between 2100 and 0200 could be classified as single birds according to the wingbeat pattern, and $3 \%$ were unclassified and flew at airspeeds below $5 \mathrm{~m} / \mathrm{s}$. Thus, the contamination of these density measurements by single large insects was low. Details on the recording and processing of migration and meteorological data are provided in Bruderer et al. (1995).

Statistics.-For each night and height interval, we calculated a flight range according to the E model and the EW model. Height distributions of flight ranges were taken as an index of suitability for the expected height distribution of the birds. Therefore, we obtained two theoretical height distributions based on predicted flight ranges for each night. We used Spearman rank correlation to compare height distributions between the two models and to compare these distributions with the height distribution of migration density. Likewise, the tailwind component per height interval, as calculated by the T model, was correlated with the altitudinal profile of migration density. No significance levels are given, because the predicted values within a height distribution are not independent (they are based on height profiles of weather data, which are autocorrelated). To test whether one model prediction was better than the other, we determined which predicted distribution was more highly correlated with the migration data and examined the resulting frequencies of superiority with a $\chi^{2}$ test. Correlation coefficients are referred to as $r$ for Pearson parametric correlations and $r_{s}$ for Spearman rank correlations. Multiple regressions were calculated with forward stepwise and backward methods using STATISTICA software.

\section{RESULTS}

Model predictions for flight range.-Predictions of flight ranges were very similar for Willow Warblers and Golden Orioles (E model, $r=$ 0.98; EW model, $r=0.89 ; n=2,997$ for both). Therefore, only data from Willow Warblers are presented. Two representative examples for the altitudinal distributions of the data analyzed are given for a single night in autumn (13 September 1992; Fig. 1A) and spring (21 April 1992; Fig. 1B). The weather data on the right side of the figures were used as input data for the flight-range predictions on the left side of the figures. A multiple regression analysis showed that flight ranges according to the $\mathrm{E}$ model were explained by tailwinds and crosswinds to a high degree $\left(R_{\text {adj }}^{2}=0.93, n=2,927\right.$, $P<0.001)$. Temperature and pressure also were significantly correlated with predictions from the E model, but the increase in explained variance was only $0.7 \%$. Flight ranges from the EW model also were dominated by tailwinds and crosswinds, but only $70 \%$ of the variance was explained by these two factors. Including pressure, temperature, and relative humidity reduced the unexplained variance to less than $20 \%\left(R_{\text {adj }}^{2}=0.84, n=2,927, P<0.001\right)$. The correlation between the predictions of these two models was also high $\left(R^{2}=0.66\right)$.

Model predictions for flight altitude.-First, we compared altitudes from the predicted maximum flight ranges and altitudes with the best tailwind component with altitudes of maximum migration density for each night. Predictions from the E model coincided most frequently with altitudes of maximum migration density (23\%; Fig. 2). All model distributions were significantly correlated with altitudes of migration densities (E model, $r=0.49$; EW model, $r=0.36$; T model, $r=0.41)$. The correlations did not differ significantly between each other (i.e. they were within the 95\% confidence limits of each other).

In a second analysis, we compared altitudinal distributions of flight range ( $E$ and $E W$ models) and tailwind components ( $\mathrm{T}$ model) with the altitudinal distribution of migration densities. The average correlation coefficient over all nights $(n=150)$ was $r_{s}=0.57$ for the $\mathrm{E}$ model, $r_{s}=0.60$ for the EW model, and $r_{s}=0.47$ for the T model. Predictions for autumn migration were much better than those for spring (Fig. 3). In general, correlations for nights with low migration densities were weaker than those when many birds were migrating. Therefore, calculating means weighed by migration density resulted in higher mean correlations ( $\mathrm{E}$ model, $r_{s}=0.64 ;$ EW model, $r_{s}=0.67 ;$ T model, $\left.r_{s}=0.55\right)$.

Although the EW model had the highest cor- 

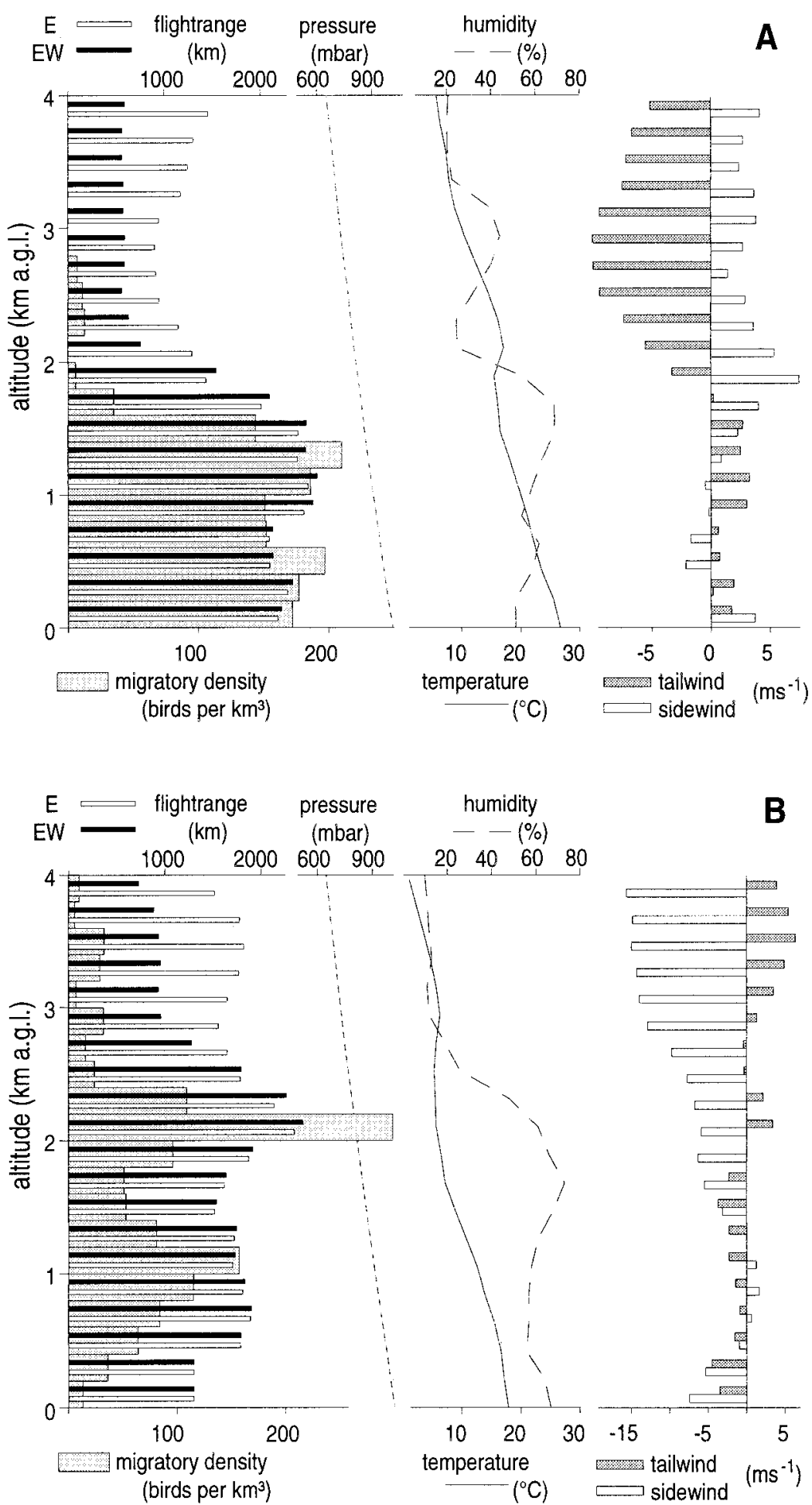

FIG. 1. Autumn (A) and spring (B) examples of the altitudinal distributions of migratory density, predictions of flight ranges according to the energy model (E) and the energy-water model (EW), and weather parameters from which model predictions were calculated (air pressure, air temperature, relative humidity, tailwind, and crosswind components). 


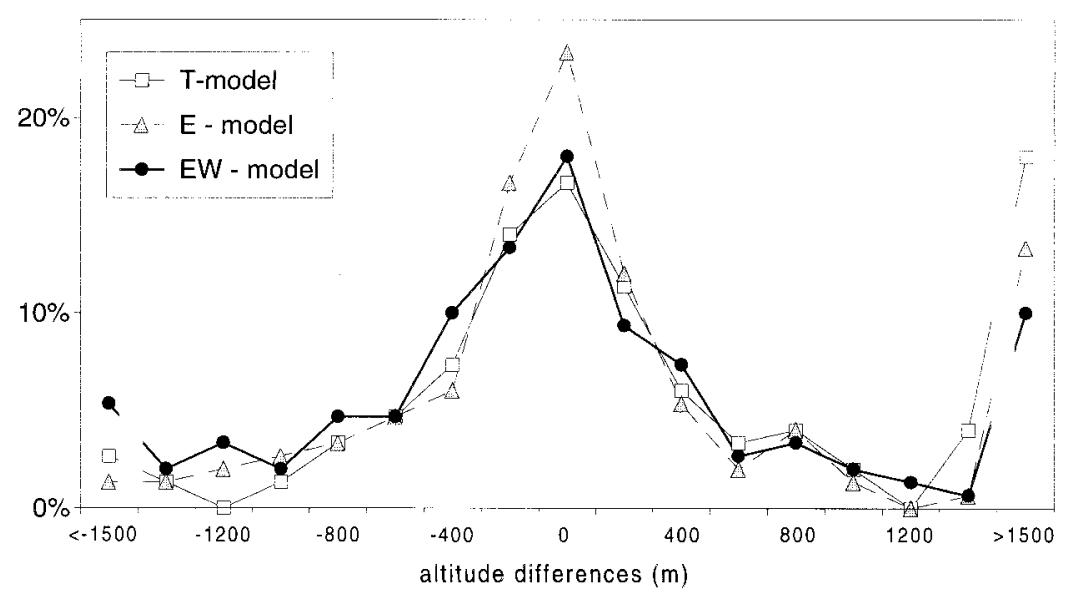

FIG. 2. Frequency distributions of the difference between predicted altitude for maximum flight range and the altitude of maximum migration density for each night. Altitudes for maximum flight range were predicted according to three alternatives: the EW model, the E model, and the T model.

relation with migration density in several cases, the frequency of superiority was not significantly different between the E and EW models (Table 1). During spring, correlations based on tailwind predictions were superior less often than those for the E and EW models (Table 1). A separate analysis of nights with low and high migratory density $\left(<20\right.$ and $\geq 20$ birds per $\left.\mathrm{km}^{3}\right)$ resulted in similar relative frequencies.

Predictions of the E and EW models were highly correlated on many nights (Fig. 4). How-

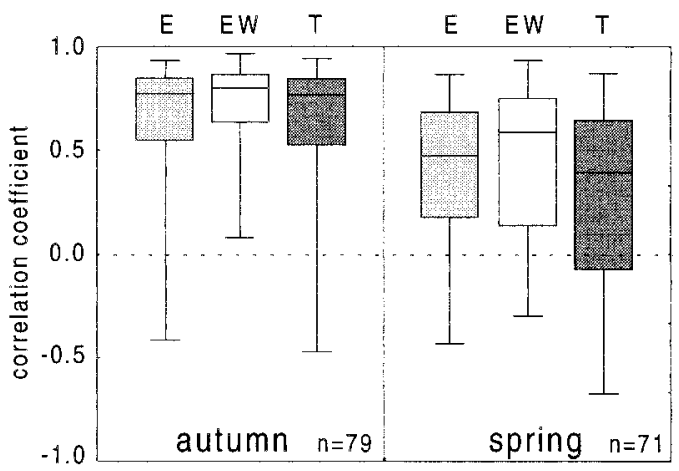

FIG. 3. Distributions of correlation coefficients between the altitudinal profiles of model predictions (EW model, E model, and T model) and the measured migration density for each spring and autumn night. Given are the median (horizontal line within box), 25 to $75 \%$ range (box), and 1 to $99 \%$ range (whiskers). Sample sizes are the number of nights per season over which the calculations were conducted. ever, there was no indication that on nights with low correspondence between the models, one or the other model was more highly correlated with migration density.

\section{DisCUSSION}

Important temporal and spatial variation in wind conditions dominated the predictions of all three models. The differences in the pre-

TABLE 1. Frequencies of the superiority of one model over the other based on correlation coefficients $\left(r_{s}\right)$ between model predictions and migration density for single nights. Significance is tested versus random occurrence of superiority.

\begin{tabular}{|c|c|c|c|c|c|}
\hline \multirow[b]{2}{*}{ Season } & \multicolumn{3}{|c|}{$\begin{array}{l}\text { Frequency of } \\
\text { superiority }\end{array}$} & \multirow[b]{2}{*}{$\chi^{2}$} & \multirow[b]{2}{*}{$P$} \\
\hline & $\begin{array}{c}\text { For } \\
\text { first } \\
\text { model }\end{array}$ & $\begin{array}{c}\text { For } \\
\text { second } \\
\text { model }\end{array}$ & $\begin{array}{l}\text { Equal } \\
\text { cases }\end{array}$ & & \\
\hline \multicolumn{6}{|c|}{ Energy model versus energy-water model } \\
\hline Autumn & 30 & 43 & 6 & 2.32 & 0.128 \\
\hline Spring & 30 & 38 & 3 & 0.94 & 0.332 \\
\hline Total & 60 & 81 & 9 & 3.13 & 0.077 \\
\hline \multicolumn{6}{|c|}{ Energy model versus tailwind } \\
\hline Autumn & 36 & 39 & 4 & 0.12 & 0.729 \\
\hline Spring & 53 & 15 & 3 & 21.24 & $<0.001$ \\
\hline Total & 89 & 54 & 7 & 8.57 & $<0.010$ \\
\hline \multicolumn{6}{|c|}{ Energy-water model versus tailwind } \\
\hline Autumn & 44 & 32 & 3 & 1.90 & 0.167 \\
\hline Spring & 53 & 18 & 0 & 17.25 & $<0.001$ \\
\hline Total & 97 & 50 & 3 & 19.03 & $<0.001$ \\
\hline
\end{tabular}




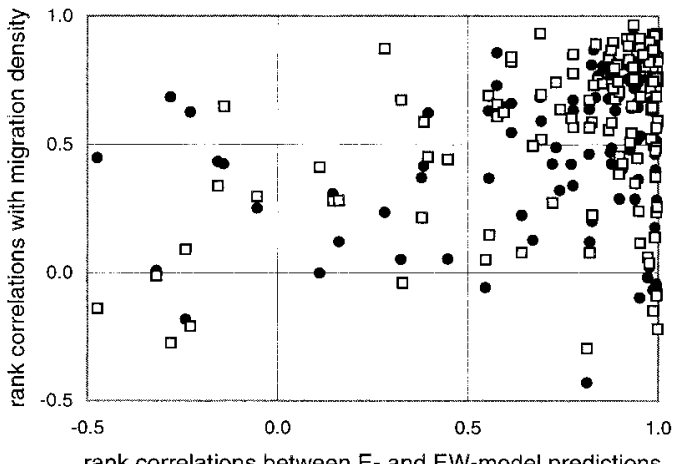

rank correlations between $\mathrm{E}$ - and $\mathrm{EW}$-model predictions

FIG. 4. Rank correlations for single nights between the energy (E) and energy and water (EW) model predictions in relation to the rank correlations between migration density and energy model predictions (black dots) and the energy and water model predictions (open squares).

dicted flight ranges and height distributions were not significant. The simple tailwind (T) model was only slightly inferior to the energy (E) model, which in turn was slightly inferior to the energy-water (EW) model.

The outcome of the EW model was not related to the various environmental conditions in a simple fashion. However, oxygen partial pressure (determined by air pressure and thus altitude) is a major determinant of respiratory water loss. Thus, in addition to migrating when tailwinds are favorable, migrants should fly at low altitudes from a water economy viewpoint. Carmi et al. (1992) encountered important differences in maximal flight ranges between the two models, but they did not take wind assistance into account. At low altitudes $(500 \mathrm{~m}$ above sea level), large differences in flightrange predictions $(>10 \%)$ occurred only if air temperature exceeded $25^{\circ} \mathrm{C}$ and relative humidity was below $30 \%$. In our weather data, only $5 \%$ of the measurements fell within this extreme range. In contrast, wind conditions that increased or decreased flight range by $10 \%$ corresponded to a change in the tailwind component of 1 or $2 \mathrm{~m} / \mathrm{s}$, which was the average variation between two neighboring height intervals of $200 \mathrm{~m}$. At 2,000 m above sea level, water balance reduced flight range by more than $10 \%$ if temperature was above $10^{\circ} \mathrm{C}$ and relative humidity was below $50 \%$, which was true for $19 \%$ of the autumn data (when wind was opposing at this altitude) and for only $6 \%$ of the spring data (when wind was usually favorable at this altitude). Thus, only in a minority of cases did the necessity to maintain water balance influence the selection of flight altitude. In the Libyan desert at low altitudes where winds were favorable for migration, Klaassen and Biebach (2000) found that the combination of temperature above $25^{\circ} \mathrm{C}$ and relative humidity below $30 \%$ occurred only rarely during autumn. In temperate latitudes with lower temperatures, higher humidity, and more variable winds than in trade wind zones, the altitudinal distribution of nocturnal migrants probably is governed by wind. However, at these latitudes, high-altitude migration ( 3 to $5 \mathrm{~km}$ ) under conditions of low oxygen partial pressure may be hampered by water loss resulting from increased pulmonary ventilation.

The energy model is rather simple in that the outcome of the model relies highly on wind conditions. Pressure provides an additional influence; for example, with a decrease in pressure (i.e. an increase in altitude) the air density declines. A reduction in air density results in decreased parasite and profile drag and increased induced drag (Pennycuick 1989). These changes result in a rather small increase in maximum range speed and a reduction of flight costs in terms of energy per unit distance (which holds only if maximum power output is not reached, a limit that may be reached by very large birds [Klaassen et al. unpubl. data]). Thus, in addition to migrating with favorable tailwinds, from an energetic viewpoint migratory birds should fly at high altitudes. Neglecting this effect of altitude (= air density) increases the predictive value of the energy model. The simplified energy model (Es model) takes into account only wind but assumes optimal adaptation of flight behavior with respect to constant track direction (Liechti et al. 1994). This resulted in correlations that were similar to the EW model $\left(r_{s}=0.59\right.$ vs. 0.60 and $r_{s}=0.67$ vs. 0.67 if weighed for migrant density). Also, the slight difference in superiority of the EW model over the E model (Table 1) disappeared (in 77 to 70 cases, the Es model was superior to the EW model). Because the Es model predicted slightly lower flight altitudes than the E model, its predictions were slightly better and approached those of the EW model. Predicting lower flight altitudes may compensate for other limitations in altitude caused, for example, by 


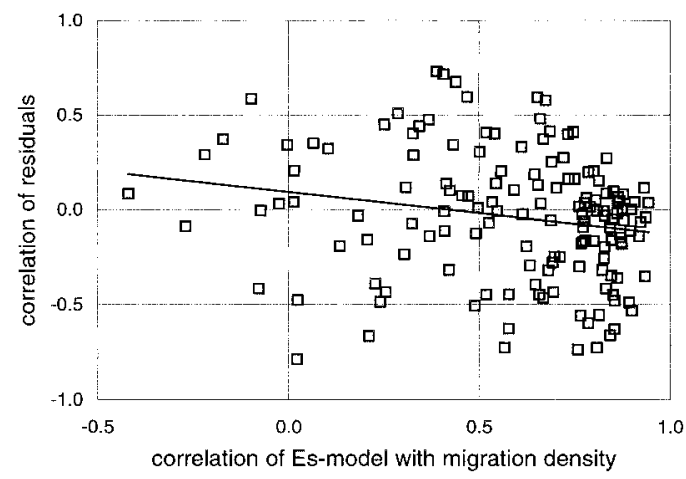

FIG. 5. Additional predictive value for the altitudinal distributions by the EW model with respect to the Es model. Residuals were calculated from linear regressions between the EW model and the Es model and also between the Es model and migration density. The $y$-axis shows the correlation coefficients of these residuals, and the $x$-axis represents the correlation coefficients between the Es model and migration density. The regression line $(y=-0.2239 x+$ 0.0944; $\left.R^{2}=0.0401, n=150, P<0.05\right)$ indicates that for nights with a very poor correlation between Es model predictions and migration density, the water budget explains some of the variance. When the Es model was a fairly good predictor, no influence of water balance could be found.

costs of climbing or limitations from reduced oxygen pressure (see below).

In light of the fact that only wind profiles are needed for the simplified Es model, and the model explains almost as much of the variation in flight altitude as the much more complex EW model, for the time being the most reasonable option may be to use the Es model to predict altitudinal distributions of nocturnal bird migration.

To investigate whether the water budget has an additional effect on the altitudinal distribution of migration in the Negev, we calculated the residuals of a linear regression between the Es model and migration densities and between the Es and EW models. Calculating correlations per night between these residuals resulted in a mean correlation close to zero $\left(r_{s}=0.04\right)$. Yet, a significant relationship occurred between these correlations and those between the Es model and migration density (Fig. 5). Although this is a rough comparison, it indicates that for the few nights when wind is a poor predictor of flight altitude, a migrant's water economy may become more prominent in determining flight altitude.
Of the four models, EW and Es tended to predict the lowest optimal flight altitudes and to provide the best fit to the empirical data. Three possible reasons, none of which is considered by any of the models, also may result in relatively low flight altitudes. First, all birds have to start and end their flights at ground level, leading to a downward bias in the apparent altitudinal distribution. This is particularly important when trade wind conditions (i.e. northerly winds below and southerly winds above altitudes of ca. $1.5 \mathrm{~km}$ ) suggest that no birds should fly below the wind shear in spring. Second, the cost of ascending may cause birds to favor low altitudes with moderately good but not optimal conditions when the benefit from ascending to higher altitudes with better conditions is small. However, for a Willow Warbler climbing at $1 \mathrm{~m} / \mathrm{s}$, it takes about half an hour to reach an altitude of 2,000 m, which will cost it a corresponding flight distance of about $7 \mathrm{~km}$ (estimated from Hedenström and Alerstam 1992). Thus, a tailwind of $1 \mathrm{~m} / \mathrm{s}$ would compensate for this energetic cost within a flight of about $2 \mathrm{~h}$. Because the energetic costs of climbing increase with size, for larger birds the difference in wind assistance between altitudes should be higher to make a climb to altitudes with more favorable winds worthwhile. Last, the efficiency of converting energy stored in body tissue into mechanical power might change with environmental conditions. Thus, oxygen provisioning may become a problem at high altitudes where oxygen partial pressure is low. In addition, compensation for wind drift based on visual cues on the ground becomes more difficult with increasing altitude. Again, because of these problems, birds may tend to fly lower than predicted by the models.

Such behavioral effects are included in the simulation of Bruderer et al. (1995), in which all birds start at ground level and are supposed to sample the tailwind vector in neighboring altitudinal zones of $200 \mathrm{~m}$, adjusting their flight levels continuously when conditions are preferable to those at previous altitudes. If the simulation is run for an infinite number of iterations, the outcome corresponds to the T model used in the present paper; if the simulation is stopped after 50 iterations in autumn and after 100 iterations in spring, the predictive power of this model is higher than that of the present models. However, the simulation is not based 


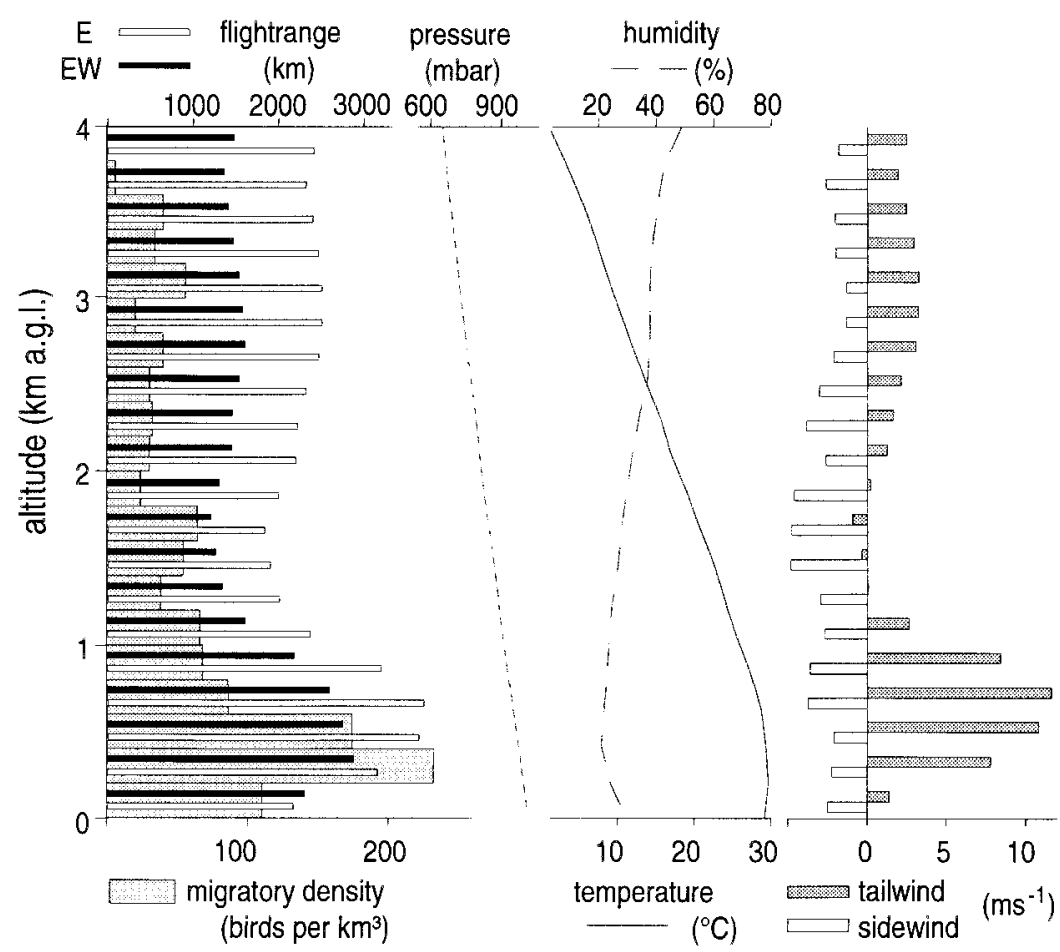

FIG. 6. An extreme example (with respect to temperature, migration altitude, and preferred flight altitude) of the altitudinal distributions of variables analyzed in this study. Data are for the night of 2 October 1991. Shown are density of migrants, predictions of flight ranges according to the energy model (E) and the energywater model (EW), and weather parameters (air pressure, air temperature, relative humidity, tailwinds, and crosswinds) from which the model predictions were calculated.

on aerodynamic and physiological arguments, but simply on the comparison of actual altitudinal distributions of birds and altitudinal wind profiles.

Not treated in the EW model is the possibility of heat stress and its effect on flight altitude. During heat stress, evaporative water loss of birds increases dramatically. Birds are therefore expected to go to great lengths to avoid heat stress, i.e. to fly at colder temperatures and thus at higher altitudes than predicted by the models. In wind-tunnel experiments, Nachtigall (1990) found critical temperatures for pigeons to be around $5^{\circ} \mathrm{C}$, whereas Kvist et al. (1998) reported $22^{\circ} \mathrm{C}$ as the critical value for Thrush Nightingale (Luscinia luscinia). Surprisingly, $15 \%$ of the migrants in our study flew when air temperatures exceeded $25^{\circ} \mathrm{C}$. This occurred mainly in autumn at low altitudes. Figure 6 shows a peak migration night in autumn 1991 when most of the migrants flew at altitudes where air temperature was about $30^{\circ} \mathrm{C}$ and relative humidity was about $25 \%$. The EW model prediction for this night was clearly better $\left(r_{s}=0.85\right)$ than that of the E model $\left(r_{s}=\right.$ $0.60)$, perhaps due to the effect of higher pressure reducing respiratory ventilation and thus respiratory water loss (despite slightly higher temperature and lower humidity). These birds did not seem to be heat stressed, because they could have flown higher at lower temperatures.

Our results show that including the water budget in a model in general does not increase the accuracy of the predictions. The model calculations show that with respect to water budget alone, a bird can fly more than 1,000 km under almost any weather conditions, which is more than it flies within a single night. Birds may choose to fly under conditions where they will dehydrate if they expect to be able to compensate for a developing water deficit farther en route. This rehydration may then be realized very quickly by drinking after landing. Landing next to potential drinking sites (small 
creeks and ponds) was observed in homing pigeons followed by helicopter on flights across high mountain ridges and/or on very hot days (Wagner 1970). Birds that cross the Negev desert on their northward flight in spring have a higher probability of finding water in northern Israel than do autumn migrants that approach the Sinai and the Sahara. Therefore, we expect that spring migrants would be more willing to fly under suboptimal conditions with regard to water balance when they are approaching better habitat than would autumn migrants that are flying toward deteriorating habitat. This expectation is corroborated by the fact that on most autumn nights, migration density decreases rapidly after midnight, whereas in spring the decline is much slower (Bruderer and Liechti 1995). The present comparison of various methods to predict the altitudinal distribution of nocturnal migrants confirms that the altitudinal wind profile is the simplest and best predictor.

\section{ACKNOWLEDGMENTS}

We are grateful to all who helped gather data in Israel. Special thanks to Thomas Steuri for developing the hardware and software for the recording equipment and the main programs for data analysis. Along with Dieter Peter, he also kept the recording system working during the observation periods. The Swiss Army provided the radar, and the Israeli Ministry of Communications provided funding. The company NIMBUS carried out the radiosondes during the first two observation periods, and in the last period we did radiosondes with equipment owned by the Paul Scherrer Institute at Würenlingen. We thank S. Gauthreaux, M. Kestenholz, R. Larkin, B. Naef-Daenzer, B. Pinshow, and R. Spaar for valuable comments on the manuscript.

\section{Literature Cited}

BRUderer, B. 1997. The study of bird migration by radar. Part 1: The technical basis. Naturwissenschaften 84:1-8.

BRUDERER, B., AND F. LIECHTI. 1995. Variation in density and height distribution of nocturnal migration in the south of Israel. Israel Journal of Zoology 41:477-487.

BRUDERER, B., L. G. UNDERHILl, AND F. LIECHTI. 1995. Altitude choice of night migrants in a desert area predicted by meteorological factors. Ibis 137:44-55.

Carmi, N., B. Pinshow, W. P. Porter, And J. Jaeger. 1992. Water and energy limitations on flight du- ration in small migrating birds. Auk 109:268276.

Hedenström, A., And T. Alerstam. 1992. Climbing performance of migrating birds as a basis for estimating limits for fuel-carrying capacity and muscle work. Journal of Experimental Biology 164:19-38.

JenNi, L., AND S. JenNi-EIERMANN. 1998. Fuel supply and metabolic constraints in migrating birds. Journal of Avian Biology 29:521-528.

KLAASSEN, M. 1995. Water and energy limitations on flight range. Auk 112:260-262.

KLAASSEN, M. 1996. Metabolic constraints on longdistance migration in birds. Journal of Experimental Biology 199:57-64.

KlaAssen, M., AND H. BiebACH. 2000. Flight altitude of trans-Sahara migrants in autumn: A comparison of radar observations with predictions from meteorological conditions and water and energy balance models. Journal of Avian Biology 31: in press

KlaAssen, M., A. KVIST, AND Å. Lindström. 1999. How body water and fuel stores affect long distance flight in migrating birds. In press in Proceedings of the 22nd International Ornithological Congress (N. Adams and R. Slotow, Eds.). Durban, 1998. University of Natal, Durban.

Kvist, A., M. KlaAssen, AND Å. Lindström. 1998. Energy expenditure in relation to flight speed: What is the power of mass loss rate estimates? Journal of Avian Biology 29:485-498.

LieCHTI, F., AND B. BRUderer. 1995. Direction, speed and composition of nocturnal bird migration in the south of Israel. Israel Journal of Zoology 41: 501-515.

Liechti, F., A. Hedenström, And T. Alerstam. 1994. Effects of sidewinds on optimal flight speed of birds. Journal of Theoretical Biology 170:219225.

Moreau, R. E. 1972. The Palaearctic-African bird migration systems. Academic Press, London.

NACHTIGALL, W. 1990. Wind tunnel measurements of long-time flights in relation to the energetics and water economy of migrating birds. Pages 319327 in Bird migration: Physiology and ecophysiology (E. Gwinner, Ed.). Springer-Verlag, Berlin.

PenNyCuick, C. J. 1989. Bird flight performance: A practical calculation manual. Oxford University Press, Oxford.

Pennycuick, C., M. Klaassen, A. Kvist, and A. LINDSTRÖM. 1997. Wingbeat frequency and the body drag anomaly: Wind-tunnel observations on a Thrush Nightingale (Luscinia luscinia) and Teal (Anas crecca). Journal of Experimental Biology 199:2757-2765.

RAYNER, J. M. V. 1990. The mechanics of flight and bird migration performance. Pages 283-299 in 
Bird migration: Physiology and ecophysiology (E. Gwinner, Ed.). Springer-Verlag, Berlin.

WAGNER, G. 1970. Verfolgung von Brieftauben im Helikopter. Revue Suisse de Zoologie 77:39-60. Williams, T. C., AND J. M. Williams. 1990. The ori- entation of transoceanic migrants. Pages 7-21 in Bird migration: Physiology and ecophysiology (E. Gwinner, Ed.). Springer-Verlag, Berlin.

Associate Editor: F. R. Moore 